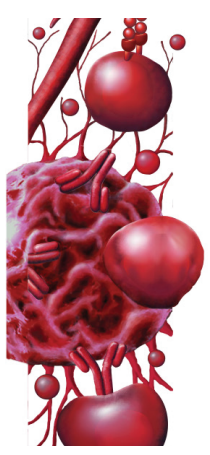

\author{
"Inflammatory processes are integral to the labor process and hence are an \\ attractive target for therapuetic intervention."
}

\title{
Are chemokines therapeutic targets for the prevention of preterm labor?
}

\begin{abstract}
Keywords: chemokines $\bullet$ inflammation $\bullet$ preterm labor $\bullet$ therapeutic $\bullet$ uterus
\end{abstract}
Preterm birth, defined as delivery less than 37 weeks gestation, remains a significant clinical and public health challenge. The rate of preterm delivery in the USA has remained around $12 \%$ in recent years, with $3.4 \%$ delivering less than 34 weeks [1]. Premature infants face immediate concerns of cardio-respiratory immaturity, but also risks of long-term neurocognitive delay and cerebral palsy. These babies account for $35 \%$ of deaths in the first year of life in the USA [2]. Approximately a quarter of babies delivered prematurely are planned deliveries indicated by medical complications, but most deliver as a result of spontaneous preterm labor or rupture of membranes. Although advances in neonatal care have improved the overall survival of preterm babies in recent years, therapeutic advances in the prevention and treatment of preterm labor have had much less impact.

The pathogenesis of preterm labor remains incompletely understood and likely represents an endpoint of multiple influencing factors, including infection, inflammation, uterine distension, cervical weakness, stress and poor nutrition. Strategies to manage preterm labor have focused first on screening and prevention, and second on treatment of the symptoms of preterm labor, hoping to stop or delay the delivery. In women identified as high risk, the two most commonly used preventative strategies are cervical cerclage and progesterone. Cervical cerclage involves insertion of a suture around the cervix in an attempt to mechanically prevent dilation. This is subject to procedural risks including rupture of membranes and miscarriage but is only moderately successful, with a meta-analysis showing a relative risk of 0.7 (95\% CI: 0.55-0.89) for cerclage over no treatment [3]. Progesterone is a key hormone involved in the control of human parturition, as it is anti-inflammatory and maintains myometrial quiescence. A recent Cochrane review concluded that progesterone treatment reduced the risk of delivery in less than 34 weeks (relative risks between 0.31 and 0.64 ) but little is known about longterm outcomes [4] and there remains debate about mechanism of action, dose and mode of administration. Once a women presents with symptoms of preterm labor, tocolytic drugs are used to reduce uterine activity. These drugs act on the pathogenic endpoint of smooth muscle contraction thus are of minimal benefit in stopping the labor altogether. Instead, they delay delivery long enough to administer corticosteroids to improve neonatal lung function [5]. Thus, there is an unmet need for effective therapeutic options for the prevention and treatment of preterm labor.

To develop novel therapies, we need to better understand the mechanisms triggering labor; this has been the subject of much recent research, with inflammatory processes being a major focus. That inflammatory mediators play important roles in labor is well established, with key roles for prostaglandins in promoting myometrial contractions and cervical ripening determined in the 1980s. More recently, genome-wide transcriptomic studies have demonstrated the magnitude and complexity of the inflammatory processes occurring in the cervix, myometrium and fetal membranes during term and preterm labor, identifying specific mediators of inflammatory processes (including cytokines, chemokines
Clare L Tower ${ }^{*, 1,2}$, Sylvia Lui $i^{1,2}$ \& Rebecca L Jones ${ }^{1,2}$ 'Maternal \& Fetal Health Research Centre, Faculty of Medical \& Human Sciences, Institute of Human Development, University of Manchester, Manchester, M13 9WL, UK

${ }^{2}$ Manchester Academic Health Science Centre, St Mary's Hospital, Central Manchester University Hospital NHS Foundation Trust, Manchester, M13 9WL, UK

*Author for correspondence: Tel.: 01612765460

clare.tower@manchester.ac.uk 
and proteases) and upstream regulators (e.g., NFKB or toll-like receptors) [6-8]. Comparative studies of animal models have ascertained strong similarities existing in the inflammatory pathways between species, despite distinct differences in the upstream drivers of the onset of parturition. This lends support to the hypothesis that inflammatory processes are integral to the labor process and hence are an attractive target for therapeutic intervention.

In particular, chemokines have been proposed to be a potential target to suppress inflammation in gestational tissues. Chemokines are a family of cytokines which act via G-protein coupled receptors (CCR and CXCR) to chemoattract and activate specific leukocyte subsets and hence orchestrate leukocyte trafficking during inflammation. Leukocyte infiltration of the cervix, myometrium and decidua occurs during labor [9,10]. Studies in rodents demonstrate immune cell infiltration occurs prior to the onset of labor, and thus is likely to be a contributor to, rather than a consequence of, labor [9]. These immune cells (primarily macrophages and neutrophils) are a source of inflammatory mediators and thus have the potential to initiate and/or augment inflammatory reactions. Targeted and unbiased array methods have been employed to identify the chemokines regulating these labor-associated inflammatory events. IL-8 or CXCL8, a classic neutrophil chemoatttractant and MCP-1 or CCL2, a monocyte chemokine, have been widely studied, and both are strongly upregulated in human myometrium, decidua, fetal membranes and cervix during labor [7,11]. Other chemokines strongly expressed in uterine tissues during labor (at term or preterm) include CCL3, -5, -8, -18 and CXCL1, -6, -9, -10 [6,8,12]. Together these chemokines would be capable of recruiting uterine immune cells and thus may be instrumental for the initiation of labor.

\section{"Chemokine redundancy suggests that a broad spectrum approach may be needed in the modification of inflammation."}

Many investigators have sought to clinically exploit the strong association of chemokines with term/preterm labor, primarily as diagnostic tools. Several chemokines (including IL-8, CCL2 and CCL3) are elevated in amniotic fluid during term and preterm labor, indicating their potential as predictors of imminent delivery. Similarly, measurement of maternal serum levels of CCL5 and IL-10, together with ultrasound assessment of cervical length, accurately predicted delivery within 7 days in women with threatened preterm labor, with a positive likelihood ratio of 5.8 and sensitivity and specificity of 74 and $87 \%$, respectively [13]. Identification of women in true preterm labor is important in the clinical management of women with threatened preterm labor, enabling therapies to be targeted to those most likely to benefit from intervention, while reducing unnecessary hospital admissions and preventing exposure of mother and fetus to unnecessary and potentially harmful drugs (e.g., tocolytics, corticosteroids).

Whether blockade of chemokine action, and consequent prevention of uterine leukocyte infiltration, has therapeutic potential for inhibiting the labor process in women with preterm labor, has not yet been tested. The potential role in other inflammatory disorders, including autoimmune diseases, multiple sclerosis, HIV and cancer has long been of interest. Two approaches exist: blockade of individual chemokine receptors or broader spectrum inhibition. Individual receptor blockade has met with limited clinical success, largely due to lack of efficacy rather than unwanted side effects. A recent review lists more than 40 chemokine receptor antagonists subjected to Phase I or II trials, but only 2 have reached clinical practice [14], largely due to lack of efficacy. Chemokine receptors CCR5 are coreceptors for HIV entry into CD4 T cells; antagonism of these receptors effectively prevents HIV infection of CD4 cells. A CCR5 inhibitor has now been licensed for use in this context [15], although CCR5 inhibition proved unsuccessful in the treatment of rheumatoid arthritis or multiple sclerosis. CXCR4 has multiple actions, one of which is retention of hematopoietic cells in bone marrow. CXCR4 inhibition therefore increases the mobilization of cells from bone marrow into the circulation, which is of interest in autologous stem cell transplantation [16].

It is notable that the clinical success of individual chemokine inhibition to date has not been via modification of inflammation. The numerous reasons for this include trial design, disease heterogeneity, but also chemokine redundancy. Chemokine redundancy suggests that a broad spectrum approach may be needed in the modification of inflammation. One such broad spectrum inhibitor, FX125L, has proved successful in Phase I studies for chronic lung inflammatory disorders, highlighting the potential of these drugs [17].

There are few studies specifically of chemokine inhibition in preterm labor. In an in vitro model involving mechanically stretched human myometrial cells, broad-spectrum chemokine inhibitor reduced leukocyte migration [18]. The same group showed the efficacy of broad-spectrum chemokine inhibitor in blocking the onset of labor in a mouse model of infection-induced preterm labor [19], although this was ineffective in stopping noninfectious preterm labor. This is likely due to the coincident activation of other inflammatory pathways, as predicted by transcriptomic studies of laboring uterine tissues, and it is possible that upstream modulation may be required for 
a more global effect on multiple pathways. Moreover, recent murine studies have shown the timing of labor is unaffected by the absence of uterine neutrophils, suggesting nonessential roles for these cells [20]. Whether macrophages, the most abundant cell type infiltrating the uterus [9], are obligatory for labor has not been tested.

\section{Conclusion}

The therapeutic option of chemokine inhibition for the prevention and treatment of preterm labor remains an attractive one. Chemokines are certainly very abundant in gestational tissues and have a strong association with labor and preterm labor. However, they represent only one aspect of a highly complex network involved in the control of inflammation within utero-placental tissues in pregnancy. The redundancy observed between chemokines and their receptors perhaps suggests that individual chemokine blockade will be challenging, with broader spectrum approaches offering more possibility of success. These latter drugs may be more likely to have additional unwanted affects. A more detailed understanding of the processes involved in the initiation of preterm labor will almost certainly aid progress toward clinically effective agents in the future.

\section{Financial \& competing interests disclosure}

The Manchester Maternal and Fetal Health Research Centre is supported by funding from Tommy's the Baby Charity, an Action Research Endowment Fund, the Manchester Biomedical Research Centre and the Greater Manchester Comprehensive Local Research Network. The authors have no other relevant affiliations or financial involvement with any organization or entity with a financial interest in or financial conflict with the subject matter or materials discussed in the manuscript apart from those disclosed.

No writing assistance was utilized in the production of this manuscript.

\section{References}

1 Martin JA, Osterman MJ. Preterm births - United States, 2006 and 2010. MMWR Surveill. Summ. 62 (Suppl. 3), 136-138 (2013).

2 Matthews TJ, Macdorman MF. Infant mortality statistics from the 2010 period linked birth/infant death data set. Natl Vital Stat. Rep. 62(8), 1-26 (2013).

3 Berghella V, Rafael TJ, Szychowski JM, Rust OA, Owen J. Cerclage for short cervix on ultrasonography in women with singleton gestations and previous preterm birth: a metaanalysis. Obstet. Gynecol. 117(3), 663-671 (2011).

4 Dodd JM, Jones L, Flenady V, Cincotta R, Crowther CA. Prenatal administration of progesterone for preventing preterm birth in women considered to be at risk of preterm birth. Cochrane Database Syst. Rev. 7, CD004947 (2013).

5 Haas DM, Caldwell DM, Kirkpatrick P, Mcintosh JJ, Welton NJ. Tocolytic therapy for preterm delivery: systematic review and network meta-analysis. BMJ 345, e6226 (2012).

6 Mittal P, Romero R, Tarca AL et al. Characterization of the myometrial transcriptome and biological pathways of spontaneous human labor at term. J. Perinat. Med. 38(6), 617-643 (2010).

7 Haddad R, Tromp G, Kuivaniemi H et al. Human spontaneous labor without histologic chorioamnionitis is characterized by an acute inflammation gene expression signature. Am. J. Obstet. Gynecol. 195(2), 394 e391-e324 (2006).

8 Hassan SS, Romero R, Haddad R et al. The transcriptome of the uterine cervix before and after spontaneous term parturition. Am. J. Obstet. Gynecol. 195(3), 778-786 (2006).

9 Hamilton SA, Oomomian Y, Stephen G et al. Macrophages infiltrate the human and rat decidua during term and preterm labour: evidence that decidual inflammation precedes labour. Biol. Reprod.(2011) (In Press).

Osman I, Young A, Ledingham MA et al. Leukocyte density and pro-inflammatory cytokine expression in human fetal membranes, decidua, cervix and myometrium before and during labour at term. Mol. Hum. Reprod. 9(1), 41-45 (2003).

11 Shynlova O, Lee YH, Srikhajon K, Lye SJ. Physiologic uterine inflammation and labor onset: integration of endocrine and mechanical signals. Reprod. Sci. 20(2), 154-167 (2013).

12 Hamilton SA, Tower CL, Jones RL. Identification of chemokines associated with the recruitment of decidual leukocytes in human labour: potential novel targets for preterm labour. PloS ONE 8(2), e56946 (2013).

13 Tsiartas P, Holst RM, Wennerholm UB et al. Prediction of spontaneous preterm delivery in women with threatened preterm labour: a prospective cohort study of multiple proteins in maternal serum. BJOG 119(7), 866-873 (2012).

14 Pease J, Horuk R. Chemokine receptor antagonists. J. Med. Chem. 55(22), 9363-9392 (2012).

15 Lieberman-Blum SS, Fung HB, Bandres JC. Maraviroc. a CCR5-receptor antagonist for the treatment of HIV-1 infection. Clin. Ther. 30(7), 1228-1250 (2008).

16 Broxmeyer HE, Orschell CM, Clapp DW et al. Rapid mobilization of murine and human hematopoietic stem and progenitor cells with AMD3100, a CXCR4 antagonist. J. Exp. Med. 201(8), 1307-1318 (2005).

17 Fox Group Research. www2.warwick.ac.uk/fac/sci/chemistry/research/fox/ foxgroup/fx1251/.

18 Lee Y-H, Shynlova O, Lye S. Chemokine receptor antagonism inhibits transendothelial migration by activated leukocytes: a potential novel approach for preterm birth prevention. Reprod. Sci. 21(3[S]), 110A (2014). 
19 Shynlova O, Dorogin A, Li YQ, Lye T, Lye S. Broad spectrum chemokine inhibitor delays infection-induced preterm delivery in mice. Reprod. Sci. 19(S3), 104A (2012).
20 Rinaldi SF, Catalano RD, Wade J, Rossi AG, Norman JE. Decidual neutrophil infiltration is not required for preterm birth in a mouse model of infection-induced preterm labor. J. Immunol. 192(5), 2315-2325 (2014). 